\title{
An Optoelectromechanical Tactile Sensor for Detection of Breast Lumps
}

\author{
Mehmet Ayyildiz, Burak Güçlü, Mustafa Zahid Yildiz, and Cagatay Basdogan
}

\begin{abstract}
We developed a compact tactile imaging ( $\mathrm{TI})$ system to guide the clinician or the self-user for noninvasive detection of breast tumors. Our system measures the force distribution based on the difference in stiffness between a palpated object and an abnormality within. The average force resolution, force range, and the spatial resolution of the device are $0.02 \mathrm{~N}, 0-4 \mathrm{~N}$, and $2.8 \mathrm{~mm}$, respectively. To evaluate the performance of the proposed TI system, compression experiments were performed to measure the sensitivity and specificity of the system in detecting tumor-like inclusions embedded in tissue-like cylindrical silicon samples. Based on the experiments performed with 11 inclusions, having two different sizes and two different stiffnesses located at three different depths, our TI system showed an average sensitivity of $90.8 \pm 8.1$ percent and an average specificity of $89.8 \pm 12.7$ percent. Finally, manual palpation experiments were performed with 12 human subjects on the same silicon samples and the results were compared to that of the TI system. The performance of the TI system was significantly better than that of the human subjects in detecting deep inclusions while the human subjects performed slightly better in detecting shallow inclusions close to the contact surface.
\end{abstract}

Index Terms-Medical imaging, tactile sensor, breast tumor detection

\section{INTRODUCTION}

$\mathrm{A}$ CCORDING to the statistics reported in 2008, breast cancer is the most common type of cancer among women, with an estimated 1.38 million new cases ( 23 percent of all the cancers) and the second most common cancer worldwide in both sexes (11 percent of all the cancers) [1]. If a breast tumor is limited to few cell layers and does not invade and destroy surrounding tissues or organs, it is considered benign (noncancerous). In contrast, if the tumor spreads to surrounding tissues or organs, it is considered malignant (cancerous) [2], [3]. Detection of breast cancer at its early stage of onset is crucial and highly dependent on the performance of the method used for breast cancer screening and diagnosis. Breast self-examination (BSE), clinical breast examination $(\mathrm{CBE})$, mammography, magnetic resonance imaging (MRI), and ultrasonography are currently the most commonly used methods for screening and diagnosis of breast cancer. However, none of these methods satisfies all the desired specifications: high sensitivity, acceptable specificity, good accuracy, ease of use, acceptability to the population being screened (considering discomfort and time), and low cost.

BSE is regular examination of breasts by women via palpation. It is a costless and noninvasive procedure for early detection of abnormalities. However, its effectiveness highly depends on the examiner's proficiency. Even if the examiner is skilled, it is often difficult for humans to detect

- M. Ayyildiz and C. Basdogan are with the College of Engineering, Koc University, Rumelifeneri Yolu 34450, Sariyer, Istanbul, Turkey.

E-mail: \{mayyildiz, cbasdogan\}@ku.edu.tr.

- B. Güçlü and M.Z. Yildiz are with the Institute of Biomedical Engineering, Boğaziçi University, Kandilli Campus, Cengelkoy, Istanbul 34684, Turkey. E-mail: \{burak.guclu, mustafa.yildiz\}@boun.edu.tr.

Manuscript received 4 Nov. 2011; revised 11 June 2012; accepted 3 July 2012; published online 10 Sept. 2012

Recommended for acceptance by A. Okamura.

For information on obtaining reprints of this article, please send e-mail to: toh@computer.org, and reference IEEECS Log Number TH-2011-11-0088.

Digital Object Identifier no. 10.1109/ToH.2012.54. small size tumors less than $1 \mathrm{~cm}$ in diameter [4]. Also, BSE does not allow one to differentiate between tumor types or provide quantitative and objective information about the tumors. $\mathrm{CBE}$ involves regular examination of the breast by a health professional. Similar to BSE, CBE requires the examiner's proficiency. An annual CBE is suggested for women older than 40 years of age [5]. Many physicians express a low confidence in their CBS skills [6], [7], which results in increased number of investigations, unnecessary biopsies, and false diagnoses [7], [8]. In addition, CBE and the associated reporting procedures are not standardized and consistent. The average size of tumors detected by CBE is $2.1 \mathrm{~cm}$ in diameter [9].

Mammography is the most commonly used breast screening modality today. It involves compressing the breast tissue between two plastic plates and applying lowdose X-rays. Unlike BSE and CBE, mammography requires specific medical equipment and supplies (a dedicated X-ray machine, radiographic film, and developing chemicals), a trained technologist to use the machine, and a radiologist to interpret the X-ray films. Especially, the compression causes considerable discomfort to patient. The mean radiation dose in mammography is approximately $4-5 \mathrm{mGy}$, but the applied dosage varies with the breast density [10]. As the dose increases, the risk of further breast cancers due to the radiation increases [11]. For this reason, mammography is not recommended for women under the age of 30 because the incidence rate of breast cancer for that group is low and their breast densities are high. Also, screening by mammography is not applicable for women who are pregnant or have breastfed within the last year.

MRI is a breast cancer screening modality that uses a powerful magnetic field and radio waves to produce images of the body. MRI is good at imaging 1) dense breasts of younger women, 2) breasts containing implants, which are often a problem for mammography owing to possible leak in the implant or rupture due to the squeezing, and 3) smaller 
lesions often missed by mammography. MRI also helps to determine the stage of breast cancer. However, MRI requires the patient to lie down for half an hour to an hour and half without moving, which can be uncomfortable [12]. MRI is not only long but is also a costly technique. Moreover, identifying false positives in MRI is a problem, and it is difficult to differentiate between benign and malignant tumors [13].

Ultrasound refers to sound waves with a frequency above the audible range of human hearing, $20 \mathrm{kHz}$. There are several modes of ultrasound and most common ones are B-mode and Doppler mode. B-mode ultrasonography uses emitted and reflected sound to create an image of the breast. It is frequently used as a follow-up test for the assessment of mammographically or clinically detected breast masses to obtain supplemental information. Also, it is used to characterize lesions of women who cannot undergo mammography owing to pregnancy or young age [14]. Since the purpose of the ultrasonography is the examination of a suspicious area in the breast, diagnosing the whole breast with ultrasonography is labor-intensive and operator-dependent [15]. Also, examination techniques are not standardized and the interpretation of the results shows variations. In Doppler sonography, the distribution and morphology of blood vessels in the breast tissue is evaluated to detect breast tumors. The Doppler effect is used to calculate the frequency shift so that the speed and the direction of the flow can be determined and visualized [16], [17].

Elastography and microwave imaging are other methods for breast cancer imaging. In elastography, stiffness contrast between the healthy and the cancerous tissue is detected. In microwave imaging, the permittivity and conductivity contrast between the healthy and the cancerous tissue is used to detect and characterize tumors in the breast tissue [18].

Tactile imaging (TI) is one of the emerging noninvasive medical imaging techniques that are used to detect tumors inside the breast tissue. Typically, TI involves applying compression to the breast tissue with a probe having an array of pressure sensors at the tip to measure the pressure distribution at the contact area. TI can estimate the shape, size, and location of a tumor by comparing its stiffness with the surrounding healthy tissue. Young's modulus measurements of breast specimens showed that healthy tissue has a significantly lower elastic modulus than that of cancerous tissue [19], [20], [21]. Benign and cancerous tumors in the tissue have distinguishable material and geometric properties as reported in [22] and [23]. TI is a relatively new method for breast examination; hence, the number of commercial devices available for clinical use is limited. Medical Tactile Inc., (Los Angeles, CA) produced a TI device under the trade name of SureTouch [24]. The system includes a probe, a processor unit, and a computer. The probe consists of $192(16 \times 12)$ pressure sensors covering an area of $40 \mathrm{~mm} \times 30 \mathrm{~mm}$. The processor unit takes 20 measurements per second and then transfers the measured data to a computer through a USB interface [25]. Using SureTouch, Kaufman et al. [26] examined 110 patients with breast masses and estimated the geometrical and material properties of these masses from the recorded tactile images. Using the same TI system, Egorov and Sarvazyan [25] conducted experiments with a silicon model and with patients having breast tumors. In their experiments, the examination was performed in two consecutive steps: 1) a general examination by linear sliding of the probe, and then 2) a local examination by making circular motions. If a suspicious area was detected in the first step, then a more detailed examination was performed in the second step. To process the collected tactile images, several signal processing techniques were used: low-pass noise-filtering, 2D noise-removal, background subtraction, signal thresholding, pixel neighborhood rating, subsampling, and 2D interpolation. They used Max/Base > noise criterion (i.e., the maximum pressure recorded for an inclusion divided by the base pressure of the surrounding tissue being greater than some predefined threshold value) and a neural network algorithm to detect the tumors. Assurance Medical Corp. (Hopkinton, MA) [27] developed a TI system consisting of a hand-held probe with $416(16 \times 26)$ piezo-resistive pressure sensors, an electromagnetic position tracker, and a data acquisition unit to sample the signals. The pressure sensors have a range of $0-34 \mathrm{kPa}$. The data acquisition unit acquires data from the tracker and the pressure sensor at $200 \mathrm{~Hz}$. The tracker records the relative position and rotation of the probe to help with the construction of a 3D tactile map of the breast being examined. The voice commands guide the clinician to help her/him exert the desired pressure to the breast tissue for reducing the variations between images [27]. Wellman and Howe [28] proposed inverse models to estimate the size and the shape of a tumor in breast tissue based on the pressure distribution recorded by this device. They stated that their forward and inverse algorithms provided accuracy at least twice as good as either CBE or ultrasonography. Yegingil et al. [29] developed a piezoelectric finger (PEF) that consists of a driving piezoelectric actuator at the top, a sensing electrode at the bottom, and a stainless steel layer in the middle. PEF could both apply force and sense the corresponding displacement simultaneously. Elastic modulus, shear modulus, and Poisson's ratio of soft polymer samples were measured via indentation and consequently, elastic and shear moduli maps were constructed in 2D. The depth and elastic modulus of an inclusion in a polymer sample were determined by using two PEFs and an empirical model made of two springs. Omata et al. [30] developed a TI system consisting of an array of 64 sensors for the examination of breast stiffness. Each sensor includes driving and sensing PZT ceramic elements and a vibration rod with a spherical tip. The elasticity of an object is estimated based on the shift in the resonance frequency of the sensor when the rod contacts the object.

\section{Methods and Materials}

\subsection{Design of Our TI System}

Our TI system consists of 100 integrated sensor elements (QRD1313, Reflective Object Sensor; Fairchild Semiconductor) arranged in a $10 \times 10$ array as shown in Fig. 1a. The sensor elements are powered by an external, regulated power supply (5 V DC). The emitter of a QRD1313 is an IR 


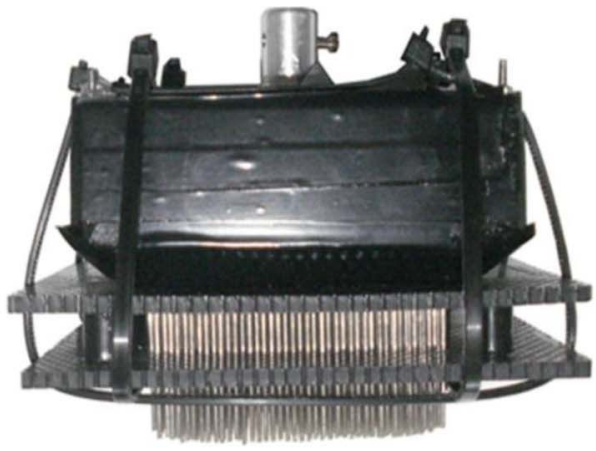

Fig. 1. (a) Our tactile sensor. (b) Cross section of our tactile sensor.

light-emitting diode (LED), and the detector is a photodarlington transistor, molded in a plastic case with a filter allowing mostly IR light rays to pass through. The sensor elements are soldered on a double-sided printed-circuit board and housed in a prismatic aluminum casing with a square base, as illustrated in Fig. 1b. The side length and the height of our TI sensor-array head are 9.2 and $3.0 \mathrm{~cm}$, respectively. The array of sensor elements is located in a $7 \mathrm{~cm} \times 7 \mathrm{~cm}$ area. The force range and the resolution of each sensor element are $0-4 \mathrm{~N}$ and $0.02 \mathrm{~N}$, respectively. The spatial resolution of the system (including the moving pins) is $2.8 \mathrm{~mm}$. A histogram of the force values measured 100 times at the center pixel using our TI system is shown in Fig. 2 . The sensor elements are covered by a silicon-rubber membrane and optically isolated from each other by a spacer grid. Also, a tactile interface consisting of two perforated covers and $25 \times 21$ moving pins is attached on the top of the rubber membrane. The perforated covers are separated $10 \mathrm{~mm}$ away from each other and the holes of the covers are aligned (see Fig. 1b). The moving pins are free to slide in independently toward the infrared emitter-detector sensors. However, they are not allowed to slide out of the perforated covers. During palpation, the palpated object interacts with the array of moving pins and hence, the moving pins slide in toward the sensor elements causing the rubber membrane to deflect due to the transmitted interaction forces. In our design, the silicon rubber membrane is fixed at the edges of the casing and its outside surface is painted black to block ambient light and to reduce

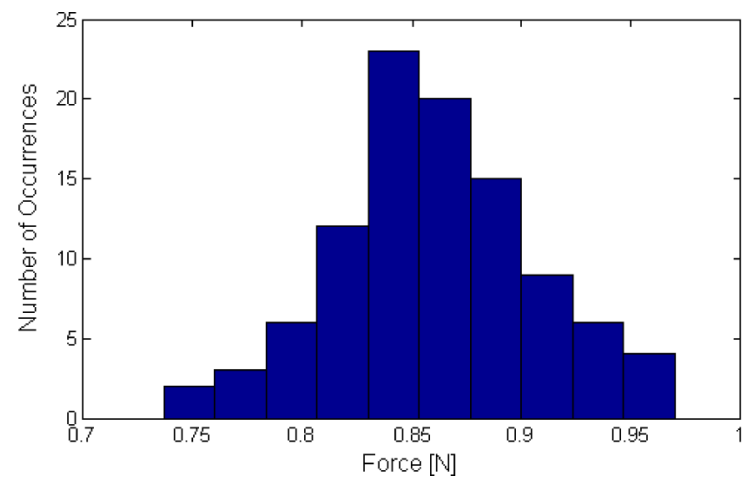

Fig. 2. A histogram of the force values measured at the center pixel for the compression depth of $19 \mathrm{~mm}$. The mean force, the standard deviation, and the coefficient of variation are $0.86 \mathrm{~N}, 0.05 \mathrm{~N}$, and 5.8 percent, respectively. stray light falling upon the phototransistors. This design of the membrane limits the deflections as well as the conformation of the palpated object by the tactile pin interface. The maximum deflection in the silicone rubber membrane is $7 \mathrm{~mm}$. When the rubber membrane is deflected, the IR light rays emitted from the LEDs reflect off from the white colored inner surface of the membrane and reach to the detectors. This reflection is modulated by the deflection of the membrane, which is caused by the force transmitted to the membrane's outer surface by the palpated object through the pin interface [31], [32]. Consequently, the light intensity in each isolated sensor element is measured to construct the tactile map of the palpated object.

In data acquisition, the light intensities measured by the IR detectors as voltage values are first transmitted to the processor unit via a flat cable and then to a 16-bit analogto-digital converter (ADC) card (NI6034, National Instruments) via a single analog channel and a synchronization channel based on the time-division multiplexing method. In this method, 10 data points from each sensor element are acquired at each multiplexing cycle, which contains data from each successive sensor ordered in time. Since the sampling rate of the ADC card is $100 \mathrm{kHz}$, one multiplexing cycle (the output from the entire array) takes $0.01 \mathrm{~s}$ $(0.01 \mathrm{~ms} \times 100$ sensors $\times 10$ data points $)$ and hence the actual scan rate of the hardware system is $100 \mathrm{~Hz}$. However, the scan rate achieved by the software is $33 \mathrm{~Hz}$ due to further signal processing explained below. The flow of the measured signal from the sensors to the ADC card connected to a PC is shown in Fig. 3. The processor unit amplifies the multiplexed data and applies offset shifting to the signal to match the input sampling range of the ADC card. The ADC card decodes the incoming analog signal with the help of a synchronization pulse generated by the processor unit at each multiplexing cycle. The voltage

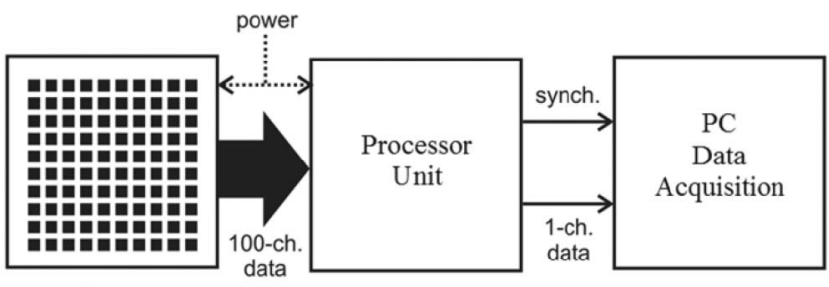

Tactile Sensor

Fig. 3. Data acquisition units of the proposed TI system. 

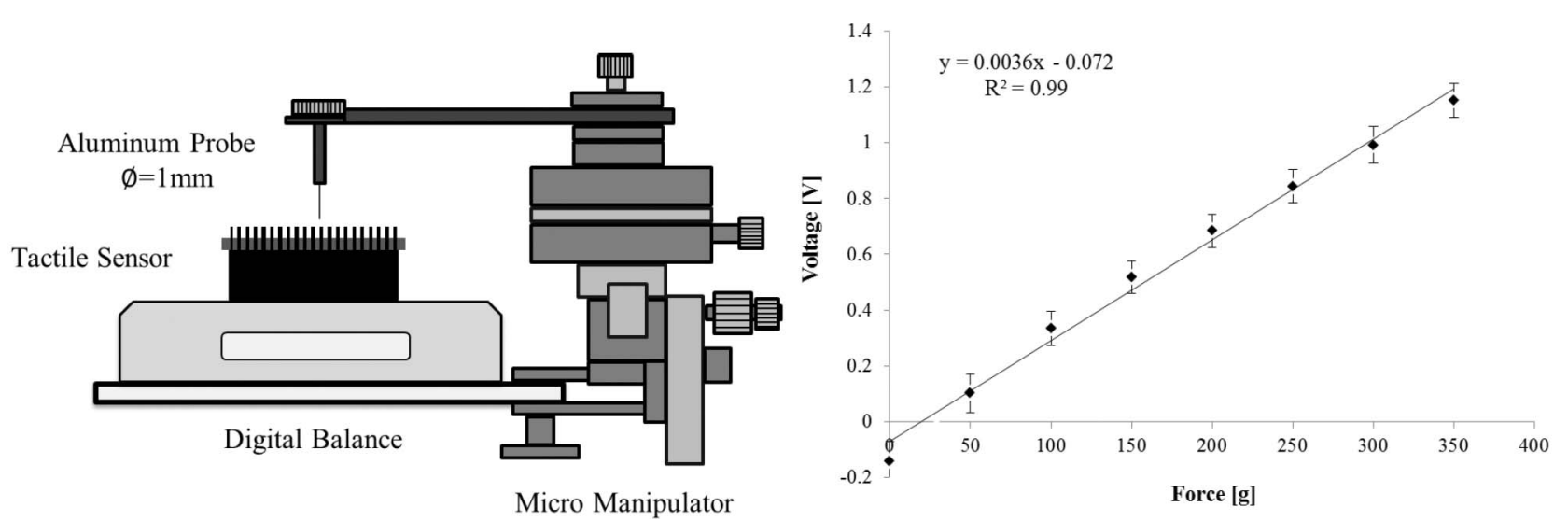

Fig. 4. (a) The calibration setup for the tactile sensor. (b) The calibration curve of a sensor element used in the TI system.

values acquired through the ADC card are converted to force values by using calibration curves.

For calibration, first, an aluminum indentation probe attached to an XYZ micromanipulator (KITE-R, World Precision Instruments, Inc., position resolution is $0.1 \mathrm{~mm}$ for all axes) is slowly pressed to each grid point on the outer surface of the silicon rubber membrane facing a sensor as shown in Fig. 4a. The voltage change in the IR detector of the sensor and the force response of the indentation probe are measured simultaneously using the data acquisition unit and a digital balance (440-49N, KERN), respectively. Hence, the calibration curve of the sensor is obtained as shown in (see Fig. 4b). This process is repeated for all the sensors in the array. The average force sensitivity and the average zero error of the tactile sensors is $0.004 \mathrm{~V} / \mathrm{g}$ and $0.094 \mathrm{~V}$, respectively. This offset voltage $(0.094 \mathrm{~V})$ is compensated later by the software. The average value of $\mathrm{R}^{2}$ for 100 sensor elements is 0.91 . (Note that approximately 10 percent of the sensors located at the edges do not show a linear behavior due to the boundary effects.)

\subsection{Data Processing}

The data processing involves four major steps: spatial interpolation, low-pass filtering, background subtraction, and thresholding (see Fig. 5).

1. Spatial interpolation. The sensor data is acquired in units of force from the $10 \times 10$ sensor array. It is spatially interpolated along the $x$ and $y$ axes to give an output force array of 100 by 100 elements.

2. Low-pass filtering. A digital FIR filter designed with a Kaiser window is used for antialiasing. The filter can achieve a stop band attenuation of $65 \mathrm{~dB}$. The spatial cutoff frequency is selected as five cycles per linear length of the tactile membrane (i.e., 0.82 cycles $/ \mathrm{cm}$ ).

3. Background subtraction. The statistical difference between the force responses of the samples in the comparison group and the control sample is tested by the Bonferroni-corrected two-sample $t$-test. The statistically different pixels are plotted as the background (control-sample pixel values) subtracted from the comparison-group pixel values.

4. Thresholding. Since the boundary sensors are more prone to artifacts and distortions in our design, additional filtering is performed on the boundary pixels. After the background subtraction, the pixel in each tactile image having the maximum force value is determined by excluding the pixels at the boundaries first. Then, the area around that pixel is determined by an edge detection algorithm. This area is defined as the suspicious area and the force values of the pixels that are outside of this area are set to zero.

\section{EXPERIMENTS}

Compression experiments were performed on tissue-like cylindrical silicon samples containing spherical silicon inclusions. The radius and the height of the cylindrical samples were 25 and $40 \mathrm{~mm}$, respectively. The spherical inclusions were prepared in two different sizes (small/ large) and in two different stiffnesses (soft/hard) and then embedded into the cylindrical samples at three different depths (shallow/intermediate/deep) during the molding process (see Fig. 6). The cylindrical sample containing the large and hard inclusion at zero depth was not used in the experiments since it was too easy to detect. The other 11 samples containing the inclusions of all other possible combinations $(2 \times 3 \times 2-1=11)$ were tested against the control sample having no inclusion.

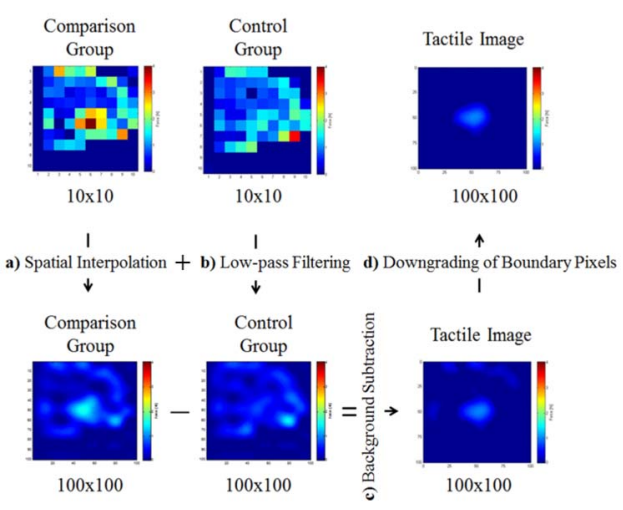

Fig. 5. The steps of our data processing: (a) the tactile images of the comparison and the control groups $(10 \times 10)$ are spatially interpolated to $100 \times 100$, (b) low-pass filtering is applied to the images for antialiasing, (c) the tactile image of the comparison group is subtracted from that of the control group, and (d) the pixels at the edges are thresholded to eliminate the boundary effects. 


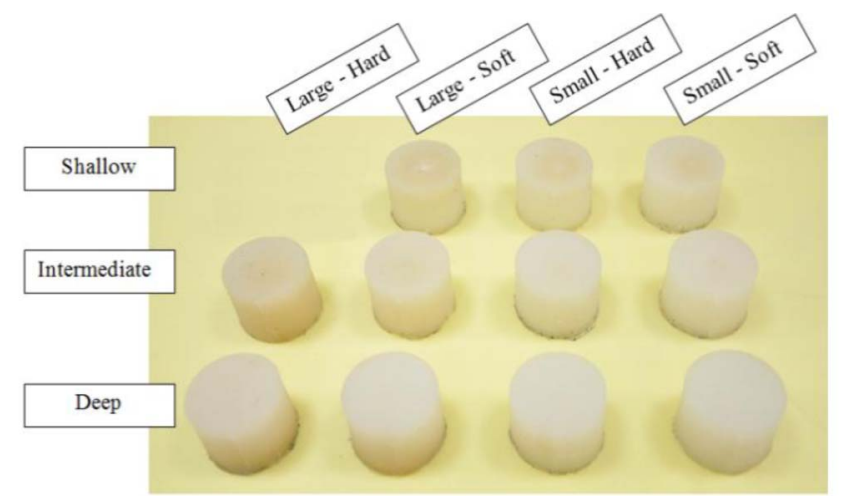

Fig. 6. The cylindrical silicon samples containing silicon inclusions.

Commercial-grade silicon (Ecoflex Supersoft 0010, Smooth-On Inc.) was used to construct tissue-like cylindrical samples. The Young's modulus of the samples was measured as 11 and $20 \mathrm{kPa}$ at 1 and 5 percent strains, respectively, using a compression setup (see Fig. 7) developed in our laboratory [33]. The tumor-like spherical silicon inclusions were prepared in two different stiffness levels using Smooth-Sil 910 (Smooth-On Inc.) commercial silicon. The Young's modulus of the soft and the hard silicon inclusions were measured as 56 and $91 \mathrm{kPa}$ at 1 percent strain and 60 and $107 \mathrm{kPa}$ at 5 percent strain, respectively. The stress versus strain curves of the samples and the inclusions are shown in Fig. 8. The inclusions were embedded into the silicon samples at $\mathrm{h}=0 \mathrm{~mm}$ (shallow),

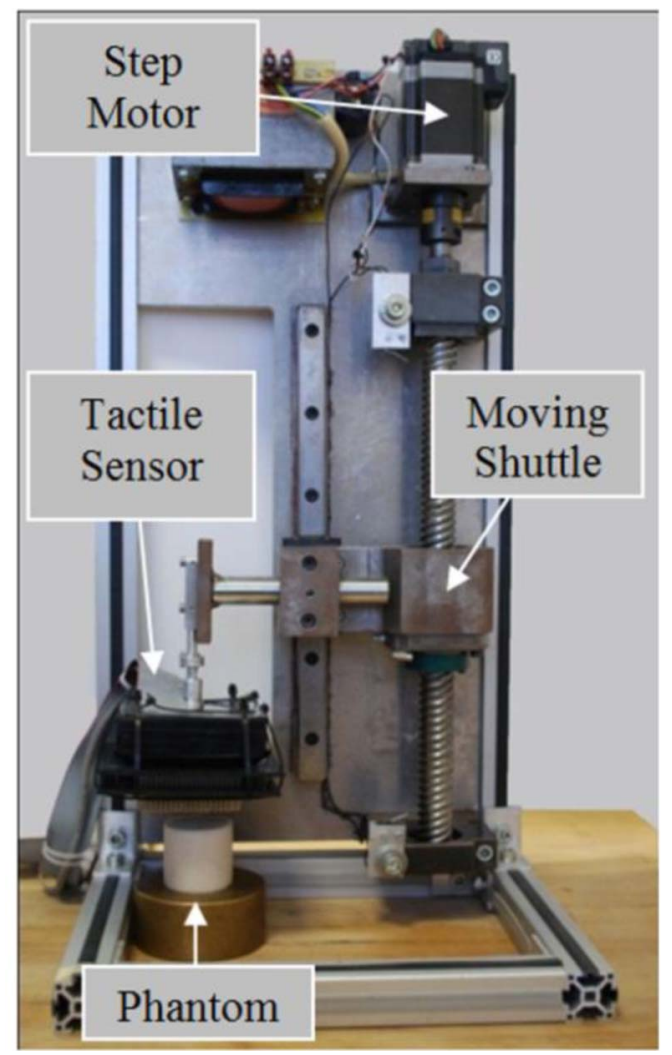

Fig. 7. The mechanical compression device used in our study to characterize material properties of the silicon samples and to evaluate the performance of our tactile sensor.

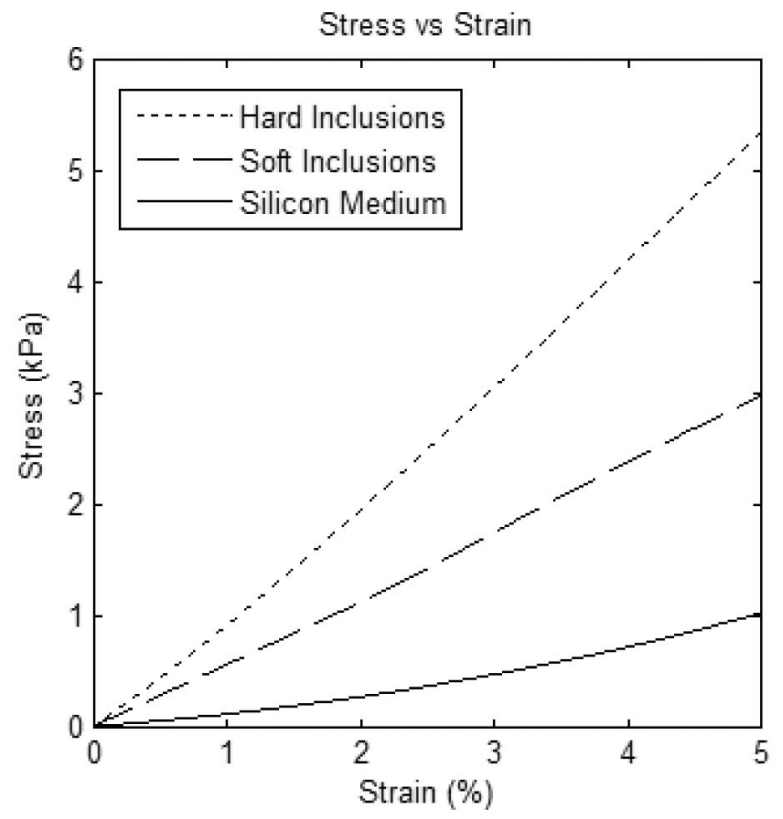

Fig. 8. The stress versus strain curve for the hard inclusion $(\mathrm{E}=91 \mathrm{kPa}$ at 1 percent strain, and $\mathrm{E}=107 \mathrm{kPa}$ at 5 percent strain), for the soft inclusion $(\mathrm{E}=56 \mathrm{kPa}$ at 1 percent strain, and $\mathrm{E}=60 \mathrm{kPa}$ at 5 percent strain), and for the tissue-like silicon medium ( $\mathrm{E}=11 \mathrm{kPa}$ at 1 percent strain, and $\mathrm{E}=20 \mathrm{kPa}$ at 5 percent strain).

$10 \mathrm{~mm}$ (intermediate), and $20 \mathrm{~mm}$ (deep) depths. The diameters of the inclusions were $\mathrm{d}=10 \mathrm{~mm}$ (small) and $20 \mathrm{~mm}$ (large). In the experiments performed on ex-vivo breast tissue samples, it was reported that fibrocystic disease and malignant tumors are up to 13 times stiffer than the breast tissue [21]. Also, breast tumors having a diameter of 10 and $20 \mathrm{~mm}$ correspond to stage 0 and stage 1 breast cancer, respectively [34]. In other studies utilizing TI to detect hard inclusions in a silicon/gelatin model, the elastic modulus of the silicone/gelatin model and the inclusions varied between $3.8-8 \mathrm{kPa}$ and $25-254 \mathrm{kPa}$, respectively, [25], [30], [35]. Also, the depth and the diameter of the inclusions used in those studies varied between 1-35 $\mathrm{mm}$ and 6-17 $\mathrm{mm}$, respectively.

\subsection{Compression Experiments}

The tactile sensor was attached to the moving shuttle of the power screw in our compression setup as shown in Fig. 7 and then advanced by the stepper motor to compress the cylindrical silicon samples slowly at a rate of $0.5 \mathrm{~mm} / \mathrm{s}$. The silicone samples containing an inclusion were compressed to the depths of 15 and $19 \mathrm{~mm}$. These depths were selected based on the results of our earlier study [36]. For each depth, the compression experiment was repeated 20 times on each silicon sample. Hence, the total number of compression experiments performed on the samples in the comparison group was 440 (2 compression depths $\times 11$ samples $\times$ 20 repetitions). In addition, 20 compression experiments were performed on the control sample for each depth. Hence, the total number of compression experiments performed on the control sample was 40 (2 compression depths $\times 1$ sample $\times 20$ repetitions). Following the experiments, the collected data was processed using the steps discussed in Section 2.2. 


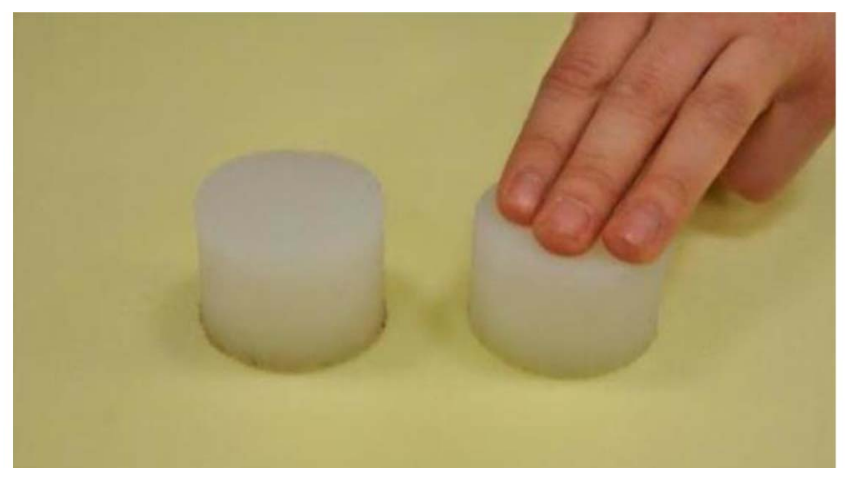

Fig. 9. The stimuli used in the manual palpation experiments.

\subsection{Performance Evaluation of our TI system}

Signal detection theory was used to evaluate the performance of the TI system. For each sample with and without an inclusion, the average force output from each sensor was recorded for the compression depths of 15 and $19 \mathrm{~mm}$. A histogram of the average forces measured by each tactile sensor was constructed for each sample. The bin width was critical in constructing the histograms: a small width led to the inclusion of unnecessary details into the histogram while a large width resulted in a coarse histogram with no details. To find the optimum bin width for each histogram, the entropy of the bin heights was maximized as suggested in [37]. To determine if a silicon sample contained an inclusion, its probability density function was compared to that of the silicon sample without any inclusion (i.e., control sample). Based on the maximum a posteriori probability rule, the optimal detection criterion was found. The null hypothesis was an empty sample, and the alternative hypothesis was a sample with an inclusion. According to the detection criterion, the areas underneath the probability density functions were found for the truenegative (TN), true-positive (TP), false-positive (FP), and false-negative (FN) probabilities. The labels, TN, TP, FP, and FN were then used to calculate sensitivity and specificity. In medical tests, sensitivity is defined as the percentage of sick people who are correctly diagnosed as having the condition (1) and specificity is the proportion of healthy people who are correctly identified as not having the condition (2). Ideally, sensitivity and specificity aim to accomplish 100 percent success so that no one is mistakenly identified as healthy or sick

$$
\begin{aligned}
& \text { Sensitivity }=\frac{\sum T P}{\sum T P+\sum F N}, \\
& \text { Specificity }=\frac{\sum T N}{\sum T N+\sum F P} .
\end{aligned}
$$

\subsection{Manual Palpation Experiments}

Manual palpation experiments were performed on the same silicon samples. Twelve naive subjects (six females and six males) participated in the experiments (Age $=25 \pm 1.5$ ). The experiment was designed with the two-alternative forced choice (2AFC) method. The subjects were asked to use the finger pads of their middle three fingers to palpate a pair of silicon samples one by one to detect the silicon sample containing an inclusion in 15 seconds (see Fig. 9). The samples were placed side by side and one of them always contained an inclusion, but its size, location, and the stiffness varied. The subjects were asked to report their decisions to the experimenter as "LEFT" or "RIGHT" depending on which sample contained an inclusion. There were 110 trials in the experiment (11 silicon pairs $\times$ 10 repetitions). The display order of the samples with inclusion and the relative location (Left/Right) of the control sample in each trial were randomized. Also, the subjects were blind-folded during the experiments to prevent any perceptual bias.

\subsection{Performance Evaluation for the Manual Palpation Experiments}

Each answer of the subjects in the manual palpation experiments resulted in either success or failure; detecting the correct sample with an inclusion (TP), which also meant rejecting the other sample without an inclusion $(\mathrm{TN}=\mathrm{TP})$ or incorrectly choosing the sample without an inclusion (FP), which also meant incorrectly rejecting the sample with an inclusion $(\mathrm{FN}=\mathrm{FP})$. For the evaluation, first the recorded responses of the subjects were labeled and then the sensitivity and specificity values were estimated from the labeled data using (1) and (2), respectively.

\section{REsULts}

\subsection{TI System}

Tactile images for the compression depths of 15 and $19 \mathrm{~mm}$ are shown in Fig. 10. The results of the statistical analysis showed that our TI system successfully detected all the embedded inclusions (see Table 1). Based on the sensitivity and the specificity values reported in Table 1 , we conclude that the tactile sensor detected the large and hard inclusions better than the small and soft ones. Also, the performance of our device was better in detecting shallow and deep inclusions than intermediate ones. It detected the deep inclusions better than the intermediate ones because deep inclusions were constrained by the lower boundary (bottom surface) of the silicon sample when the sample was compressed by the device. As a result, the forces transmitted by the inclusion to the contact interface were amplified.

\subsection{Manual Palpation Experiments}

The percentage of the false diagnoses made by the subjects with respect to the inclusion size, depth, and stiffness is shown in Fig. 11. The results show that the false diagnoses made by the subjects increased as the inclusions were placed deeper. Also, it was easier for the subjects to detect the large and stiff inclusions than the small and soft ones. More interestingly, the percentage of the false diagnoses for the hard-small inclusions was less than that of the soft-large inclusions at the deepest level. In other words, the stiffness was a more distinguishable factor than the size in detecting deep inclusions by manual palpation.

Table 1 also tabulates the average sensitivity and specificity for the human subjects in detecting inclusions by manual palpation. Since the 2AFC method was used for the design of the manual palpation experiments, the response of a subject in each trial automatically resulted 


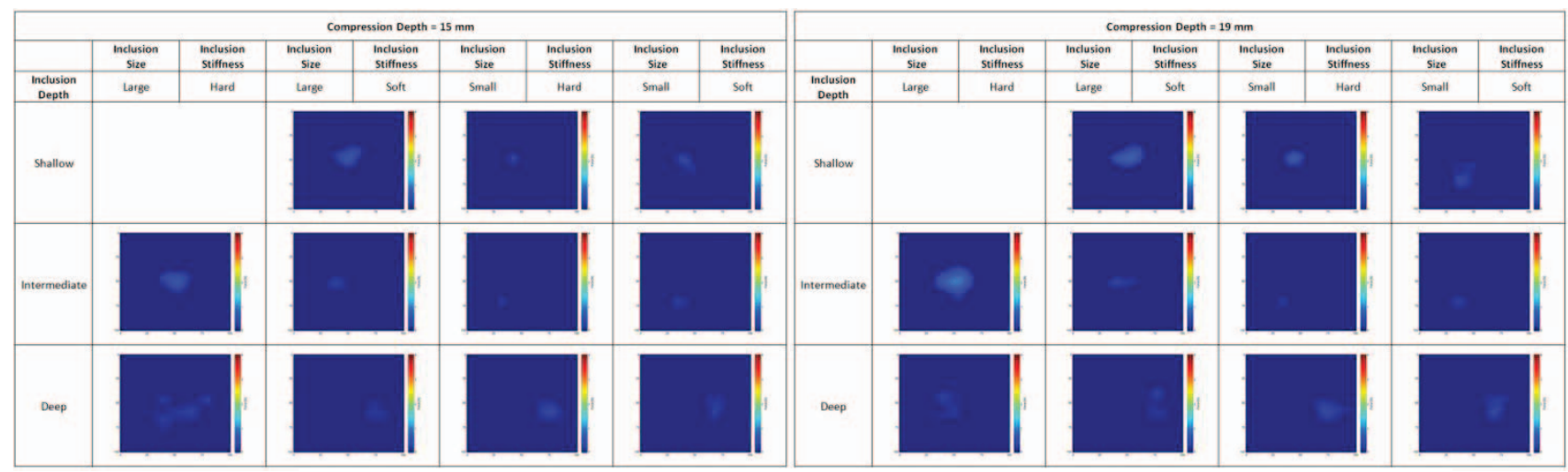

a)

b)

Fig. 10. The tactile images of the silicon samples used in our experiments for the compression depths of (a) $15 \mathrm{~mm}$ and (b) $19 \mathrm{~mm}$.

TABLE 1

The Sensitivity and the Specificity for the Tactile Sensor and the Human Palpation Including the Comparison of Them Using a Two-Sample Proportional Z-Test (Statistically Significant If Z-Score $>1.96$ or Z-Score $<-1.96$ )

\begin{tabular}{|c|c|c|c|c|c|c|c|c|}
\hline \multirow[b]{2}{*}{$\begin{array}{c}\text { Inclusion } \\
\text { Depth }\end{array}$} & \multirow[b]{2}{*}{$\begin{array}{c}\text { Inclusion } \\
\text { Size }\end{array}$} & \multirow[b]{2}{*}{$\begin{array}{c}\text { Inclusion } \\
\text { Stiffness } \\
\end{array}$} & \multicolumn{2}{|c|}{ Tactile Sensor } & \multicolumn{2}{|c|}{ Human Palpation } & \multicolumn{2}{|c|}{ Comparison } \\
\hline & & & Sensitivity & Specificity & Sensitivity & Specificity & Sensitivity & Specificity \\
\hline \multirow{3}{*}{ Shallow } & Large & Soft & $96.72 \pm 0.48 \%$ & $98.75 \pm 0.05 \%$ & $100.00 \pm 0.00 \%$ & $100.00 \pm 0.00 \%$ & $<-1.96$ & -1.23 \\
\hline & \multirow{2}{*}{ Small } & Hard & $94.21 \pm 2.02 \%$ & $94.62 \pm 5.61 \%$ & $97.22 \pm 7.40 \%$ & $97.22 \pm 7.40 \%$ & -1.11 & -0.98 \\
\hline & & Soft & $89.97 \pm 0.08 \%$ & $91.52 \pm 3.89 \%$ & $96.53 \pm 6.36 \%$ & $96.53 \pm 6.36 \%$ & $<-1.96$ & -1.59 \\
\hline \multirow{4}{*}{$\begin{array}{l}\text { Interme- } \\
\text { diate }\end{array}$} & \multirow{2}{*}{ Large } & Hard & $96.29 \pm 4.44 \%$ & $95.88 \pm 5.56 \%$ & $95.49 \pm 7.42 \%$ & $95.49 \pm 7.42 \%$ & 0.30 & 0.14 \\
\hline & & Soft & $89.66 \pm 1.81 \%$ & $92.11 \pm 3.90 \%$ & $87.85 \pm 9.31 \%$ & $87.85 \pm 9.31 \%$ & 0.42 & 1.04 \\
\hline & \multirow{2}{*}{ Small } & Hard & $78.69 \pm 5.85 \%$ & $63.21 \pm 8.37 \%$ & $67.71 \pm 26.09 \%$ & $67.71 \pm 26.09 \%$ & 1.82 & -0.70 \\
\hline & & Soft & $72.56 \pm 2.62 \%$ & $66.08 \pm 1.24 \%$ & $65.28 \pm 14.14 \%$ & $65.28 \pm 14.14 \%$ & 1.16 & 0.12 \\
\hline \multirow{4}{*}{ Deep } & \multirow{2}{*}{ Large } & Hard & $98.05 \pm 0.61 \%$ & $98.74 \pm 0.99 \%$ & $89.24 \pm 10.87 \%$ & $89.24 \pm 10.87 \%$ & $>1.96$ & $>1.96$ \\
\hline & & Soft & $92.83 \pm 0.38 \%$ & $94.67 \pm 2.86 \%$ & $65.97 \pm 17.66 \%$ & $65.97 \pm 17.66 \%$ & $>1.96$ & $>1.96$ \\
\hline & \multirow[b]{2}{*}{ Small } & Hard & $96.04 \pm 1.13 \%$ & $96.58 \pm 3.86 \%$ & $70.24 \pm 16.73 \%$ & $70.24 \pm 16.73 \%$ & $>1.96$ & $>1.96$ \\
\hline & & Soft & $94.04 \pm 0.30 \%$ & $95.62 \pm 4.60 \%$ & $39.06 \pm 16.24 \%$ & $39.06 \pm 16.24 \%$ & $>1.96$ & $>1.96$ \\
\hline
\end{tabular}

in either success, (TP and TN) or failure (FP and FN). As observed from Table 1, all the sensitivity and specificity values decreased as the depth of the inclusion was increased. In addition, these measures were higher for the silicon samples containing large and hard inclusions, indicating a better detection rate.

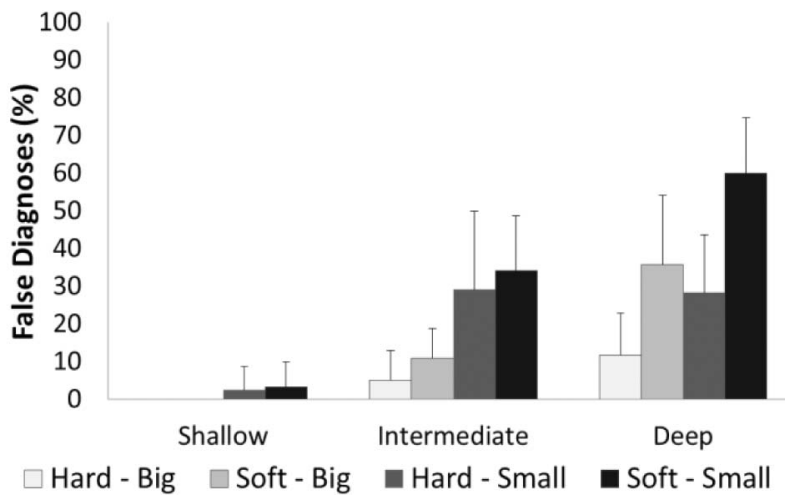

Fig. 11. The percentage of the false diagnoses made by the subjects.

\subsection{Comparison of the TI System and the Manual Palpation Experiments}

We conducted a two-sample proportional $z$-test to compare the performance of the subjects in the manual palpation experiments to that of the TI system (see Table 1). It was observed that the performance of the TI system was significantly better than that of the subjects in the manual palpation experiments for the deep inclusions while the subjects performed better in detecting shallow inclusions, except for the small-hard inclusion. There was no significant difference between the groups in detecting the inclusions at the intermediate depth. Moreover, the standard deviations of the sensitivity and specificity values for the manual palpation were higher than those for the tactile sensor.

\section{Discussion}

In this paper, we present a novel optoelectromechanical TI system, which can be utilized to guide the clinician or the self-user at home for noninvasive detection of tumors in breast tissue [36]. In our design, an array of optical elements 
consisting of an integrated IR emitting diode and a photodarlington transistor were used as sensors. These sensors detected the local deformation of a rubber membrane, which was caused by an array of pins when they were pressed on the palpated object. The rubber membrane was fixed at the edges of the casing. While, this design allowed larger deflections at the center area, it also limited the movement of the membrane at the edges and hence, resulted in greater variation in the sensor readings. Thereby, 10 percent of the sensor elements located at the edges did not show a perfect linear behavior due to the boundary effects and hence the raw values acquired from those sensors were thresholded at zero during the postprocessing. In spite of this, the average value of goodness of fit was calculated as $R^{2}=0.9128$ for 100 sensors, which indicates the overall linearity of the sensor design. The moving pins used at the contact interface reduced the cross-talk between the neighboring sensors and made the TI system more sensitive to local deformations over the rubber membrane. Nevertheless, the use of pins initially limited the spatial resolution of the TI system to $2.8 \mathrm{~mm}$, which is the distance between two adjacent pins in our current design. On the other hand, the spatial interpolation applied to the tactile images during the data processing step improved this limit by almost tenfold. Egorov and Sarvazyan [25] and Wellman et al. [27] reported that each pressure sensor in their design covered an area of $2.5 \mathrm{~mm} \times 2.5 \mathrm{~mm}$ and $1.5 \mathrm{~mm} \times 1.5 \mathrm{~mm}$, respectively. Based on this information, the spatial resolution of our system $(2.8 \mathrm{~mm})$ is lower than the spatial resolution of their systems. However, our current design utilizes large-size but low-cost sensors, which are widely available in the market. Hence, the spatial resolution of our system can be easily improved by employing smaller size but more expensive sensor elements and developing a contact interface having a denser array of pins.

To investigate the performance of the proposed TI system, we conducted compression experiments with cylindrical silicon samples containing tumor-like silicon inclusions. The results were compared to that of the manual palpation experiments performed with 12 human subjects. In our experiments, the cylindrical silicon samples contained tumor-like silicon inclusions with a diameter of 10 and $20 \mathrm{~mm}$, which corresponds to stage 0 and stage 1 breast cancer, respectively [34]. The elastic modulus of the embedded inclusions was 5-8 times stiffer than that of the control sample at 1 percent strain. In the experiments performed on ex-vivo breast tissue samples having fibrocystic disease and malignant tumors, 3-6 fold increase in stiffness has been observed while high-grade invasive ductal carcinoma has been reported to cause up to 13-fold increase in stiffness [21]. Hence, the silicon inclusions used in our experiments closely mimicked the tumors typically encountered in clinical cases.

The results of the compression experiments showed that the proposed TI system successfully detected all the tumorlike inclusions embedded into the tissue-like silicon samples. This was achieved by comparing the measured data with the reference data collected from an empty sample (i.e., control sample). The inclusion detection capability of our system is comparable to the other TI systems. In fact, it is important to emphasize that the stiffness contrast between the control sample and the tumor-like inclusions used in the other studies is higher than that of ours, which makes it easier for their devices to detect the inclusions. In our experiments, the most challenging inclusion to detect had a diameter of $10 \mathrm{~mm}$ and was located at $20 \mathrm{~mm}$ in depth. Egorov and Sarvazyan [25] prepared a silicon model containing inclusions with $6,8,11$, $14.5 \mathrm{~mm}$ in diameters. These inclusions were embedded into the model at the varying depths of $7.5-35 \mathrm{~mm}$. The elastic modulus of the control sample and the inclusions were 8 and $175 \mathrm{kPa}$, respectively. Hence, the inclusions were about 22 times stiffer than the control sample. They successfully detected inclusions, having a diameter of $11 \mathrm{~mm}$ diameter, up to $20 \mathrm{~mm}$ in depth using the Max/ Base $>$ noise criterion discussed in Section 1 and also up to $27.5 \mathrm{~mm}$ in depth using an estimation algorithm based on neural networks. In addition, smaller inclusions having $6 \mathrm{~mm}$ diameter were detected up to $10 \mathrm{~mm}$ in depth using the Max/Base > noise criterion and up to $17.5 \mathrm{~mm}$ in depth using the neural networks. Wellman et al. [27] conducted experiments with 23 subjects who underwent breast surgery. Before the surgery, the size of each breast mass was estimated by their TI system. Their TI system estimated the size of the breast masses with a mean absolute error of 13 percent while the CBE and ultrasonography yielded an error of 46 and 34 percent, respectively. Yegingil [35] used silicon inclusions inserted into a gelatin model at varying depths of 1-17 mm. The inclusions were 38 times stiffer than the gelatin model. They successfully detected the inclusions up to 8,12 , and $17 \mathrm{~mm}$ in depth using three different PEFs having different shape, size, and sensitivity. Omata et al. [30] used a silicone rubber phantom and silicone inclusions with a diameter of $13 \mathrm{~mm}$ and having three different elastic moduli: 25, 62, and $254 \mathrm{kPa}$. The inclusions were placed at depths varying from 4 to $20 \mathrm{~mm}$. The results of the experiments showed that their TI system could detect inclusions up to $20 \mathrm{~mm}$ in depth.

The performance evaluation of our TI system, quantified by sensitivity and specificity, also yielded promising results. Our TI system showed an average sensitivity of $90.82 \pm 8.08$ percent and an average specificity of $89.80 \pm$ 12.66 percent. For comparison, the sensitivity and specificity values obtained in other studies are tabulated in Table 2 . Based on this table, the use of the proposed TI system at home is justifiable since the sensitivity and specificity values reported for BSE in [38] show large variations and are lower than those of our TI system. Table 2 also shows that the performance of our TI system is also comparable to the other TI systems.

Finally, the performance of the proposed TI system was compared to that of human manual palpation. In the experiments performed with the same silicon samples, the performance of the TI system was significantly better than that of the human subjects in detecting deep inclusions while human subjects performed slightly better in detecting shallow inclusions close to the contact surface. In detecting the inclusions at the intermediate depth, there was no significant difference between the sensor and the subjects. We speculate that the receptors lying in the cutaneous 
TABLE 2

The Summary of Sensitivity and Specificity Results of Breast Cancer Imaging Modalities

\begin{tabular}{|c|c|c|c|}
\hline Modality & Reference & Sensitivity & Specificity \\
\hline BSE & Kosters et al. [38] & $26-89 \%$ & $66-81 \%$ \\
\hline CBE & Barton et al. [39] & $54 \%$ & $94 \%$ \\
\hline Mammography & Pisano et al. [40] & $70 \%$ & $92 \%$ \\
\hline MRI & $\begin{array}{c}\text { Kriege et al., Leach et al, Warner et al., Kuhl et al., Lehman et } \\
\text { al., Ely and Vioral [41-46] }\end{array}$ & $87.5 \%$ & $92.8 \%$ \\
\hline B-mode ultrasound & Scaperrotta et al. [47] & $80 \%$ & $80.9 \%$ \\
\hline Conventional ultrasound & Scaperrotta et al. [47] & $95.4 \%$ & $87.4 \%$ \\
\hline Doppler Sonography & Raza and Baum [17] & $68 \%$ & $95 \%$ \\
\hline MRE & Sinkus et al. [48] & $95 \%$ & $80 \%$ \\
\hline TI & Egorov et al. [49] & $89.4 \% \pm 7.8 \%$ & $88.9 \% \pm 7.6 \%$ \\
\hline TI & Kaufman et al. [26] & $94 \%$ & - \\
\hline TI & Yegingil [35] & $89 \%$ & $82 \%$ \\
\hline TI & This study & $90.82 \pm 8.08 \%$ & $89.80 \pm 12.66 \%$ \\
\hline
\end{tabular}

tissue of the finger pad essentially sensed the curvature of the shallow inclusions better than the TI system. However, as the inclusions were placed deeper in the samples, kinesthetic sensing of reaction forces rather than tactile sensing of curvature and pressure changes became more dominant in detecting inclusions and the TI system performed better.

\section{Conclusion}

The aim of this study was to develop a low-cost TI system, which can be used by clinicians and home users to detect tumors in breast tissue. Our initial goal was to develop a prototype system that could perform as well as manual palpation (in fact, the experiments performed with silicon samples showed that the proposed system performed better in detecting deep inclusions than manual palpation) while providing quantitative and objective data to the user. Having a TI system at home, returning quantitative data, is important for the cancer patients not only for the reasons of privacy and personal comfort, but also to record and monitor their progress regularly. Moreover, if the recorded data can be transferred to a hospital over the internet, their response to a new treatment method or a drug can be also monitored more carefully.

The proposed TI system has several benefits. Compared to the conventional imaging techniques, the proposed TI system is cost effective. Since it does not expose the patient to the hazards of radiation and powerful magnetic fields, it does not have any known side effects during or after the examination and it can potentially be used by women who are pregnant or breastfeeding. Moreover, our design does not require complex circuitry; hence, it is less prone to electrical noise compared to the capacitive systems used for tactile sensing. Also, in contrast to piezoelectricbased tactile systems, it does not require high voltages. Nevertheless, the current design is open to many improvements and optimizations. The physical size of the system can be reduced by using more compact and smaller sensor elements. Also, the resolution can be improved by increasing the number of sensors per area. In terms of usage, the device must be applied to the palpated surface using a straight downward motion and then must be lifted up before moving to a new location, hence it does not allow the user to explore the surface using sweep or drag based search patterns as in some other TI systems [25], [27], [35]. Moreover, our current system cannot estimate the size, location, and stiffness of a lump in breast tissue, compared to the same TI systems.

In the future, we would like to conduct clinical studies and test our TI system on patients having breast cancer. For this purpose, we will first miniaturize our system and increase the number of sensors per area. We will attach round tips, made of soft plastic, to the pins to make the use of device more comfortable for the patients. Furthermore, to eliminate the need for a position sensor, we will adopt a force-based approach for the measurements instead of the position-based approach used in this study. In this approach, the measurement data will be recorded only when the force applied by the user on the palpated object exceeds a certain threshold value. Moreover, the software developed for detecting inclusions from the tactile images will be further improved by implementing more advanced statistical estimation methods as suggested in some other breast imaging studies [25], [50]. Finally, a palpation procedure for the clinical examination will be designed such that the data will be collected from the healthy and unhealthy breasts of the patient for comparison. Typically, the densities of the left and right breasts of a healthy individual are highly correlated [51], [52] and taking the healthy breast as the control group should not be a problem. Obviously, any significant difference in the densities of the breasts will be judged as a tumor candidate by our current system. However, this is a useful FP that would warrant further clinical testing. Moreover, if the membrane of our tactile sensor can be extended beyond the area of the sensor array to mitigate boundary effects, then the sensors at the edges can be used as the control group as in Egorov and Sarvazyan [25]. In this way, the breasts with different size and/or shape can be examined independent of each other. However, before moving to the clinical testing on real patients, the new design and the measurement protocol will be tested on a commercial breast phantom. 


\section{REFERENCES}

[1] J. Ferlay, H.R. Shin, F. Bray, D. Forman, C. Mathers, and D.M. Parkin, "Estimates of Worldwide Burden of Cancer in 2008: GLOBOCAN 2008," Int'l J. Cancer, vol. 127, pp. 2893-2917, 2010.

[2] eHealthMD, "Breast Cancer," http://ehealthmd.com/library/ breastcancer, 2011.

[3] J. Sariego, "Breast Cancer in the Young Patient," Am. Surgery, vol. 76, no. 12, pp. 1397-1400, 2010.

[4] M. Vahabi, "Breast Cancer Screening Methods: A Review of the Evidence," Health Care Women Int'l, vol. 24, no. 9, pp. 773-793, 2003.

[5] American Cancer Society, Breast Cancer Facts and Figures 20072008. Am. Cancer Soc., 2008.

[6] C. Pilgrim, C. Lannon, R.P. Harris, W. Cogburn, and S.W. Fletcher, "Improving Clinical Breast Examination Training in a Medical School: A Randomized Controlled Trial," J. General Internal Medicine, vol. 8, no. 12, pp. 685-688, 1993,

[7] J.M. Wiecha and P. Gann, "Provider Confidence in Breast Examination," Family Practice Research J., vol. 13, no. 1, pp. 37-41, 1993,

[8] D.S. Lane and M.A. Burg, "Promoting Physician Preventive Practices: Needs Assessment for CME in Breast Cancer Detection," J. Continuing Education in the Health Profession, vol. 9, no. 4, pp. 245-256, 1989,

[9] J. Zeng, Y. Wang, M. Freedman, and S.K. Mun, "Finger Tracking for Breast Palpation Quantification with Stereo Color Cameras.," http:/ / www.simulation.georgetown.edu/spie_oe.html, 2002.

[10] K. Armstrong, E. Moye, S. Williams, J.A. Berlin, and E.E. Reynolds, "Screening Mammography in Women 40 to 49 Years of Age: A Systematic Review for the American College of Physicians," Annals of Internal Medicine, vol. 146, no. 7, pp. 516526, 2007.

[11] G.R. Howe and J. McLaughlin, "Breast Cancer Mortality between 1950 and 1987 after Exposure to Fractionated Moderate-Dose-Rate Ionizing Radiation in the Canadian Fluoroscopy Cohort Study and a Comparison with Breast Cancer Mortality in the Atomic Bomb Survivors Study," Radiation Research, vol. 145, no. 6, pp. 694707, 1996.

[12] UK CancerHelp, “MRI Scan,” http://www.cancerhelp.org.uk/ about-cancer/tests/mri-scan, 2011.

[13] Imaginis, "Scientists Find Mammography is Still 'Gold Standard' for Breast Cancer Detection but Recommend Research into More Accurate Methods," http://www.imaginis.com/breast-healthnews / scientists-find-mammography-is-still-quot-gold-standardquot-for-breast-cancer-detection-but-recomme, 2001

[14] C.M. Sehgal, S.P. Weinstein, P.H. Arger, and E.F. Conant, "A Review of Breast Ultrasound," J. Mammary Gland Biology and Neoplasia, vol. 11, no. 2, pp. 113-123, 2006.

[15] C.F. Nemec, J. Listinsky, and A. Rim, "How Should We Screen for Breast Cancer? Mammography, Ultrasonography, MRI," Cleveland Clinic J. Medicine, vol. 74, no. 12, pp. 897-904, 2007.

[16] D.O. Cosgrove, R.P. Kedar, J.C. Bamber, B. al-Murrani, J.B. Davey, C. Fisher, J.A. McKinna, W.E. Svensson, E. Tohno, and E. Vagios, "Breast Diseases: Color Doppler US in Differential Diagnosis," Radiology, vol. 189, no. 1, pp. 99-104, 1993.

[17] S. Raza and J.K. Baum, "Solid Breast Lesions: Evaluation with Power Doppler US," Radiology, vol. 203, no. 1, pp. 164-168, 1997.

[18] G.A. Ybarra, W.T. Joine, Q.H. Li, and J.P. Stang, "Emerging Technology in Breast Imaging and Mammography," Microwave Breast Imaging, J. Suri, et al., eds., pp. 1-12, Am. Scientific, 2007.

[19] A. Samani, J. Bishop, C. Luginbuhl, and D.B. Plewes, "Measuring the Elastic Modulus of Ex Vivo Small Tissue Samples," Physics in Medicine and Biology, vol. 48, no. 14, pp. 2183-2198, 2003.

[20] P.S. Wellman, R.D. Howe, E. Dalton, and K.A. Kern, "Breast Tissue Stiffness in Compression is Correlated to Histological Diagnosis," http://biorobotics.harvard.edu/pubs/1999/ mechprops.pdf, 1999.

[21] A. Sarvazyan, D. Goukassian, E. Maevsky, G. Oranskaja, G. Mironova, V. Sholokhov, and V. Ermilova, "Elasticity Imaging as a New Modality of Medical Imaging for Cancer Detection," Proc. Int'l Workshop Interaction of Ultrasound with Biological Media, pp. 69-81, 1994.

[22] A.R. Skovorda, A.N. Klishko, D.A. Gusakian, E.I. Maevskii, V.D. Ermilova, G.A. Oranskaia, and A.P. Sarvazian, "Quantitative Analysis of Mechanical Characteristics of Pathologically Altered Soft Biological Tissues," Biofizika, vol. 40, no. 6, pp. 1335-1340, 1995.
[23] T.A. Krouskop, T.M. Wheeler, F. Kallel, B.S. Garra, and T. Hall, "Elastic Moduli of Breast and Prostate Tissues under Compression," Ultrasonic Imaging, vol. 20, no. 4, pp. 260-274, 1998.

[24] Medical Tactile Inc., "SureTouch," http://www.medicaltactile. com, 2012.

[25] V. Egorov and A.P. Sarvazyan, "Mechanical Imaging of the Breast," IEEE Trans. Medical Imaging, vol. 27, no. 9, pp. 1275-1287, Sept. 2008.

[26] C.S. Kaufman, L. Jacobson, B.A. Bachman, and L.B. Kaufman, "Digital Documentation of the Physical Examination: Moving the Clinical Breast Exam to the Electronic Medical Record," Am. J. Surgery, vol. 192, no. 4, pp. 444-449, 2006.

[27] P.S. Wellman, E.P. Dalton, D. Krag, K.A. Kern, and R.D. Howe, "Tactile Imaging of Breast Masses: First Clinical Report," Archives of Surgery, vol. 136, no. 2, pp. 204-208, 2001.

[28] P.S. Wellman and R.D. Howe, "Extracting Features from Tactile Maps," Proc. Second Int'l Conf. Medical Image Computing and Computer-Assisted Intervention (MICCAI '99), vol. 1679, pp. 1133$1142,1999$.

[29] H.O. Yegingil, W.Y. Shih, W. Anjum, A.D. Brooks, and W.-H. Shih, "Soft Tissue Elastic Modulus Measurement and Tumor Detection Using Piezoelectric Fingers," Proc. Materials Research Soc. Symp., pp. 1-6. 2005,

[30] S. Omata, Y. Murayama, M. Haruta, Y. Hatakeyama, T. Shiina, H. Sakuma, S. Takenoshita, and C.E. Constantinou, "Development of a New Instrument for Examination of Stiffness in the Breast Using Haptic Sensor Technology," Sensors and Actuators A-Physical, vol. 143 , no. 2 , pp. $430-438$. 2008 ,

[31] J.G. Webster, Tactile Sensors for Robotics and Medicine. Wiley, 1988

[32] P. Polygerinos, L.D. Seneviratne, and K. Althoefer, "Modeling of Light Intensity-Modulated Fiber-Optic Displacement Sensors," IEEE Trans. Instrumentation and Measurement, vol. 60, no. 4, pp. 1408-1415, Apr. 2011.

[33] S. Ocal, M.U. Ozcan, I. Basdogan, and C. Basdogan, "Effect of Preservation Period on the Viscoelastic Material Properties of Soft Tissues with Implications for Liver Transplantation," J. Biomechanical Eng., vol. 132, no. 10, article 101007, 2010.

[34] P. Hermanek and L.H. Sobin, TNM Classification of Malignant Tumours. Springer-Verlag, 1987.

[35] H.O. Yegingil, "Breast Cancer Detection and Differentiation Using Piezoelectric Fingers," Drexel Univ., 2009.

[36] M. Ayyildiz, B. Guclu, M. Yildiz, and C. Basdogan, "A Novel Tactile Sensor for Detecting Lumps in Breast Tissue," Proc. Int'l Conf. Haptics: Generating and Perceiving tangible Sensations (EuroHaptics), pp. 367-372, 2010.

[37] B. Guclu, "Maximizing the Entropy of Histogram Bar Heights to Explore Neural Activity: A Simulation Study on Auditory and Tactile Fibers," Acta Neurobiolgiae Experimentalis (Wars), vol. 65, no. 4, pp. 399-407, 2005.

[38] J.P. Kosters and P.C. Gotzsche, "Regular Self-Examination or Clinical Examination for Early Detection of Breast Cancer," Cochrane Database of Systematic Rev., no. 2, article CD003373, 2003.

[39] M.B. Barton, R. Harris, and S.W. Fletcher, "Does This Patient Have Breast Cancer? The Screening Clinical Breast Examination: Should It be Done? How?," J. Am. Medical Assoc., vol. 282, no. 13, pp. 12701280, 1999.

[40] E.D. Pisano, C. Gatsonis, E. Hendrick, M. Yaffe, J.K. Baum, S. Acharyya, E.F. Conant, L.L. Fajardo, L. Bassett, C. D'Orsi, R. Jong, and M. Rebner, "Diagnostic Performance of Digital versus Film Mammography for Breast-Cancer Screening," New England J. Medicine, vol. 353, no. 17, pp. 1773-1783, 2005.

[41] M. Kriege, C.T.M. Brekelmans, C. Boetes, P.E. Besnard, H.M Zonderland, I.M. Obdeijn, R.A. Manoliu, T. Kok, H. Peterse, M.M.A. Tilanus-Linthorst, S.H. Muller, S. Meijer, J.C. Oosterwijk, L.V.A.M. Beex, R.A.E.M. Tollenaar, H.J. de Koning, E.J.T. Rutgers, J.G.M. Klijn, and M.R.I. Screeni, "Efficacy of MRI and Mammography for Breast-Cancer Screening in Women with a Familial or Genetic Predisposition," New England J. Medicine, vol. 351, no. 5, pp. 427-437, 2004.

[42] M.O. Leach, C.R. Boggis, A.K. Dixon, D.F. Easton, R.A. Eeles, D.G Evans, F.J. Gilbert, I. Griebsch, R.J. Hoff, P. Kessar, S.R. Lakhani, S.M. Moss, A. Nerurkar, A.R. Padhani, L.J. Pointon, D. Thompson, R.M. Warren, and MARIBS Study Group, "Screening with Magnetic Resonance Imaging and Mammography of a UK Population at High Familial Risk of Breast Cancer: A Prospective Multicentre Cohort Study (MARIBS)," Lancet, vol. 365, no. 9473, pp. 1769-1778, 2005. 
[43] E. Warner, D.B. Plewes, K.A. Hill, P.A. Causer, J.T. Zubovits, R.A. Jong, M.R. Cutrara, G. DeBoer, M.J. Yaffe, S.J. Messner, W.S. Meschino, C.A. Piron, and S.A. Narod, "Surveillance of BRCA1 and BRCA2 Mutation Carriers with Magnetic Resonance Imaging, Ultrasound, Mammography, and Clinical Breast Examination," J. Am. Medical Assoc., vol. 292, no. 11, pp. 1317-1325, 2004.

[44] C.K. Kuhl, S. Schrading, C.C. Leutner, N. Morakkabati-Spitz, E. Wardelmann, R. Fimmers, W. Kuhn, and H.H. Schild, "Mammography, Breast Ultrasound, and Magnetic Resonance Imaging for Surveillance of Women at High Familial Risk for Breast Cancer," J. Clinical Oncology, vol. 23, no. 33, pp. 8469-8476, 2005.

[45] C.D. Lehman, J.D. Blume, P. Weatherall, D. Thickman, N. Hylton, E. Warner, E. Pisano, S.J. Schmitt, C. Gatsonis, M. Schnall, and I.B.M.C. Wor, "Screening Women at High Risk for Breast Cancer with Mammography and Magnetic Resonance Imaging," Cancer, vol. 103, no. 9, pp. 1898-1905, 2005.

[46] S. Ely and A.N. Vioral, "Breast Cancer Overview," Plastic Surgery Nurse, vol. 27, no. 3, pp. 128-133, quiz 134-125, 2007.

[47] G. Scaperrotta, C. Ferranti, C. Costa, L. Mariani, M. Marchesini, L. Suman, C. Folini, and S. Bergonzi, "Role of Sonoelastography in Non-Palpable Breast Lesions," European Radiology, vol. 18, no. 11, pp. 2381-2389, 2008.

[48] R. Sinkus, K. Siegmann, M. Tanter, T. Xydeas, and M. Fink, “MR Elastography Is Capable of Increasing the Specificity of MR Mammography-Influence of Rheology on the Diagnostic Gain," Proc. Fifth Int'l Conf. Ultrasonic Measurement and Imaging of Tissue Elasticity, p. 111, 2006.

[49] V. Egorov, T. Kearney, S.B. Pollak, C. Rohatgi, N. Sarvazyan, S. Airapetian, S. Browning, and A. Sarvazyan, "Differentiation of Benign and Malignant Breast Lesions by Mechanical Imaging," Breast Cancer Research and Treatment, vol. 118, no. 1, pp. 67-80, 2009.

[50] P.S. Wellman, "Tactile Imaging," Division of Eng. and Applied Sciences, Harvard Univ., 1999.

[51] J.A. Shepherd, S. Malkov, B. Fan, A. Laidevant, R. Novotny, and G. Maskarinec, "Breast Density Assessment in Adolescent Girls Using Dual-Energy X-Ray Absorptiometry: A Feasibility Study," Cancer Epidemiology Biomarkers and Prevention, vol. 17, no. 7, pp. 1709-1713, 2008.

[52] D. Kontos, P. Bakic, R. Acciavatti, E. Conant, and A. Maidment, "A Comparative Study of Volumetric and Area-Based Breast Density Estimation in Digital Mammography: Results from a Screening Population Digital Mammography," Proc. 10th Int'l Conf. Digital Mammography, pp. 378-385, 2010

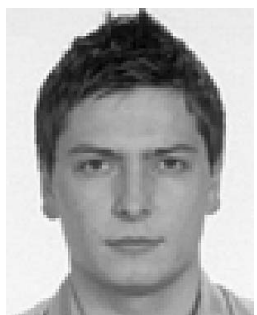

Mehmet Ayyildiz received the BSc degree with honors in mechanical engineering from Selcuk University, Turkey, in 2009 and the MSc degree in mechanical engineering from Koc University, Istanbul, Turkey, in 2011. His research interests include tactile sensing, medical imaging, haptics, biomechanics, and biomechatronics. He is currently a member of the Robotics and Mechatronics Laboratory at Koc University. He was awarded scholarships from the Scientific and Technological Research Council of Turkey to pursue his MSc and PhD studies.

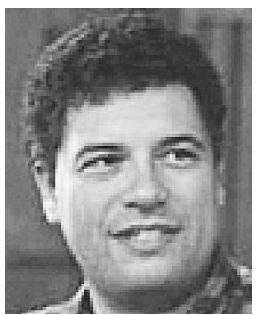

Burak Güçlü received the BS degree in control and computer engineering from the Istanbul Technical University, Turkey, in 1997, and the MS degree in bioengineering and the PhD degree in neuroscience, both from Syracuse University, New York, in 1999 and 2003, respectively, for his work on the physiology, psychophysics, and modeling of the sense of touch. During his postdoctoral research, he studied species-specific vocalizations and recorded from the prefrontal cortex of awake-behaving macaque monkeys at the medical school of the University of Rochester. He has been a faculty member of the Institute of Biomedical Engineering at Boğaziçi University since 2004, where he is also the director of the Tactile Research Lab.

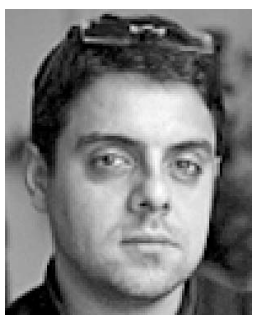

Mustafa Zahid Yildiz received the BS degree in electrical and electronics engineering from $\mathrm{Sa}$ karya University, Hendek, Turkey, in 2004 and the MS degree in biomedical engineering from Boğaziçi University, Turkey, in 2007. He is currently a member of the Tactile Research Lab at the Institute of Biomedical Engineering, Bogazici University. His research interests include psychophysics of human touch, tactile sensors, biomedical instrumentation, and measurements.

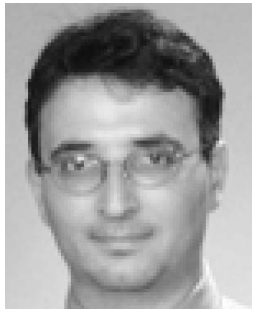

Cagatay Basdogan received the $\mathrm{PhD}$ degree in mechanical engineering from Southern Methodist University, Dallas, Texas, in 1994. He is currently a faculty member in the Mechanical Engineering and Computational Sciences and Engineering Programs at Koc University, Istanbul, Turkey, and the director of the Robotics and Mechatronics Laboratory at Koc University. Before joining Koc University, he worked at NASA-JPL/Caltech, MIT, and Northwestern University Research Park. His research interests include haptics, robotics, mechatronics, biomechanics, medical simulation, computer graphics, and multimodal virtual environments. He is currently an associate editor of the IEEE Transactions on Haptics and Computer Animation and Virtual Worlds journals. He was the general chair of the IEEE World Haptics Conference held in Istanbul in 2011.

$\triangleright$ For more information on this or any other computing topic, please visit our Digital Library at www.computer.org/publications/dlib. 